\title{
Imaging the Vessel Wall in Major Peripheral Arteries using Susceptibility Weighted Imaging
}

\author{
Qi Yang, MD11, Jiantao Liu, MD1, Samuel R.S. Barnes, MS², Zhen Wu, MD ${ }^{3}$, Kuncheng Li, \\ MD' ${ }^{1}$, Jaladhar Neelavalli, $\mathbf{P h D}^{2}$, Jiani $\mathrm{Hu}, \mathrm{PhD}^{4}$, and E. Mark Haacke, $\mathbf{P h D}^{2,5}$ \\ ${ }^{1}$ Radiology Department, Xuanwu Hospital, Capital Medical University, Beijing, China \\ ${ }^{2}$ Department of Biomedical Engineering, Wayne State University, Detroit, Michigan, USA \\ ${ }^{3}$ School of Biomedical Engineering, McMaster University, Hamilton, Ontario, Canada \\ ${ }^{4}$ Department of Radiology, Wayne State University, Detroit, Michigan, USA \\ ${ }^{5}$ The MRI Institute for Biomedical Research, Detroit, Michigan, USA
}

\begin{abstract}
Purpose-To demonstrate a novel contrast mechanism for imaging the vessel wall and vessel wall calcification using susceptibility weighted imaging (SWI).
\end{abstract}

\begin{abstract}
Materials and Methods-18 subjects were imaged with multi-detector computed tomography (MDCT) and high resolution SWI at 3T. The SWI imaging parameters were optimized to allow for the best visualization of the femoral artery lumen and the arterial wall in magnitude and phase images, respectively. SWI filtered phase data were used to evaluate the diamagnetic susceptibility of vessel wall and of putative vessel wall calcification. Imaging was performed using TE $=15.6$ $\mathrm{ms}$ (in-phase for fat); TR $=25 \mathrm{~ms}, \mathrm{FA}=10^{\circ}, \mathrm{BW}=80 \mathrm{~Hz} /$ pixel, resolution $=0.5 \mathrm{~mm} \times 0.5 \mathrm{~mm}$ inplane and $1.0 \mathrm{~mm}$ through-plane, an acquisition matrix of $512 \times 384 \times 64$ (for read, phase and slice-select directions) and a total scan time of 8 minutes.
\end{abstract}

Results-Nineteen calcifications were identified in CT and SWI and they correlated well in both size and position. The contrast-to-noise ratio between the blood signal in the lumen of the artery and arterial wall was 11.7:1 and 7.4:1 in magnitude and in phase images, respectively.

Conclusion-SWI provides a novel means to visualize vessel wall and recognize the presence of calcification.

\section{Keywords}

susceptibility; calcification; susceptibility weighted imaging; phase imaging; atherosclerosis

\section{INTRODUCTION}

Magnetic resonance imaging (MRI) has been used for many years to study atherosclerosis (1-5). Carotid arteries are the most commonly studied vessels because of their clinical significance in cerebral stroke. The sequences used in carotid artery plaque imaging have been well-tested with both bright- and black-blood methods. To date, black-blood techniques are the most ubiquitous and are used to suppress the signal from flowing blood

Corresponding Author: E. Mark Haacke, PhD, Wayne State University, MR Research Facility, Department of Radiology, HUHMR Research G030/Radiology, 3990 John R Road, Detroit, MI 48201, USA, Tel. (313) 745-1395, Fax (313) 745-9182, nmrimaging@aol.com. 
(6), making the vessel wall more conspicuous. The resulting contrast has been shown (5) to be good enough to differentiate the many types of atherosclerotic lesions according to the American Heart Association (AHA) classification scheme (7). These include: fatty plaque, fibrous plaque, vulnerable or hemorrhagic plaque, vessel wall thickening and plaque due to calcification. To distinguish between these types of atherosclerotic lesions, a multi-contrast MR protocol has been used, including T1, T2, proton-density and contrast-enhanced T1weighted sequences (8-11). It is possible to distinguish between the different types of atherosclerotic plaque by analyzing their signal intensities and morphological appearances across different imaging sequences. The black-blood approach has been the method of choice for investigating atherosclerosis. However, the black-blood approach presents two major drawbacks. The first is the potential incomplete suppression of flow (especially complex flow) which can lead to ghosting artifacts and misinterpretation of blood as vessel wall. The latter can lead to an overestimation of the vessel wall thickness (12). The second disadvantage is the fact that these methods have traditionally been two-dimensional (2D) in nature and have had a relatively long acquisition time in multislice 2D imaging. In the past, this limitation has been overcome using a 3D time-of-flight (TOF) "bright-blood" method to identify fibrous caps or intimal calcification, which manifests as a hypointense juxtaluminal band between the bright flowing blood and soft tissue $(13,14)$. Recently, some 3D SSFP techniques have been proposed that offer a more efficient 3D acquisition. While these techniques show promise, they still rely on flowing blood, which restricts the field of view (15) or requires adjustments based of the speed of the flow (16).

As a systemic disease, atherosclerosis occurs in other arteries, including the peripheral arteries. Its manifestation in the leg appears as peripheral arterial disease (PAD), affecting 8 to 10 million people in the United States alone (17). The advantage of studying atherosclerosis for femoral/popliteal arterial segments is that the leg exhibits little motion (18). Recently, researchers have tried to use the black-blood spin echo T2 methods and have had good success in imaging the wall of the femoral artery (19-21). However, the double inversion recovery and presaturation black-blood methods used have difficulties suppressing the blood signal when vessels have slower flow $(12,22)$.

Calcification is considered a major indicator of PAD. In a recent paper, it has been shown that the tibial artery calcification score is associated with the stage of the disease. Compared to traditional risk factors, this score presented a better marker to identify people who are at high risk for amputation (23). There is pathological evidence supporting the fact that calcification of arteries happens without the presence of atherosclerotic plaque (24). Finally, the American Heart Association task force designed a classification scheme for popliteal and superficial femoral artery disease to help decide whether treatment should consist of percutaneous transluminal angioplasty (PTA) or surgery $(25,26)$. They divided femoropopliteal lesions into four categories according to: 1 ) the number of segments with stenosis, 2) length of stenosis, 3) calcification, and 4) whether the vessel is completely occluded or not. If the lesion falls in category 1 or 2 , the prognosis is to do PTA. On the other hand, if the lesion falls in category 3 or 4 , bypass surgery is needed.

In this paper, we show that it is possible to image the vessel wall and calcifications with susceptibility weighted imaging (SWI) to obtain full region of interest coverage in 3D without the need to suppress the signal from the blood. SWI is a fully flow compensated sequence that aims to have no flow dependence in the signal apart from a possible TOF effect on blood signal. SWI also tends to use longer echo times to enhance the susceptibility effect between tissues. This long echo time at $3 \mathrm{~T}$ also enhances arterial contrast and suppresses venous signal because of the longer $\mathrm{T} 2 *$ of arterial blood compared to venous blood. SWI has been used in the past for imaging veins and blood products such as hemosiderin particularly to study vascular disease $(27,28)$. The changes in phase induced in 
a flow compensated gradient echo experiment such as SWI lead to both a reduction in $\mathrm{T} 2 *$ of the local tissue and to a local phase effect often visible in SWI filtered phase images. Furthermore, given the broad spectrum of plaque types (fibrous, calcified, hemorrhagic, fatty), and the fact that each may have a specific susceptibility, it is only natural to look for unique signatures of plaque using SWI. Therefore, we hypothesize: first, that the high resolution, long echo time magnitude images will show better $\mathrm{T} 2 *$ contrast for calcium and vessel wall; second, the phase images will delineate inner and outer boundaries of the vessel wall; and third, the phase images can help determine if the source of the signal change is from calcium, which is diamagnetic, versus hemorrhage, which is paramagnetic. Further, the use of magnitude and phase images separately and together leads to a means to identify calcifications unambiguously and to separate vessel wall from surrounding membranous tissue. In order to investigate the clinical utility of this approach, we studied 18 subjects with both multi-detector computed tomography (MDCT) and SWI at 3T.

\section{MATERIALS AND METHODS}

\section{Optimizing the Imaging Parameters}

Although the choice of most imaging parameters for SWI is fairly straight forward, there is some flexibility in terms of the choice of resolution (for time considerations), flip angle (for contrast in the magnitude images) and echo time (for phase contrast). First, we considered the issue of resolution. The SWI sequence we use is a conventional flow compensated gradient echo sequence. In this study, we use a single echo of $15.6 \mathrm{~ms}$ and a low bandwidth of $80 \mathrm{~Hz} /$ pixel to maximize contrast-to-noise in both magnitude and phase images. In order to image the vessel wall throughout the entire field-of-view in three dimensions, we collected the data sagittally with a high in-plane resolution of $0.5 \mathrm{~mm}$ by $0.5 \mathrm{~mm}$ and a slice thickness of $1 \mathrm{~mm}$. This has proven to be sufficient to estimate tissue properties and to identify phase differences between blood and vessel wall. Although a $0.5 \mathrm{~mm}$ isotropic resolution would be ideal, with the current sequence, this would take 16 minutes (which would lead to a reduction in SNR of $\sqrt{2}$ ). It would also be possible to keep the scanning time to 8 minutes, but this would require a reduction in the number of slices by half or the use of parallel imaging (both of which would lead to a reduction in SNR of 2). Therefore, we chose to stay with a resolution of $0.5 \mathrm{~mm} \times 0.5 \mathrm{~mm} \times 1 \mathrm{~mm}$.

Second, we considered the issue of flip angle. This sequence was applied on a healthy volunteer, with $5^{\circ}, 10^{\circ}, 15^{\circ}, 20^{\circ}$ and $25^{\circ}$ to determine the best choice of a flip angle to create the highest contrast of vessel wall versus lumen. For tissues with a T1 of $1500 \mathrm{~ms}$ (roughly $\mathrm{T} 1$ of muscle at $3 \mathrm{~T}$ ) and a TR of $25 \mathrm{~ms}$, the Ernst angle is roughly $\sqrt{(2 \mathrm{TR} / \mathrm{T} 1)}$ ) or $11^{\circ}$; while for a $\mathrm{T} 1$ of $2000 \mathrm{~ms}$ (roughly $\mathrm{T} 1$ of blood at $3 \mathrm{~T}$ ), it is roughly $9.5^{\circ}$. Given the slowly varying signal around the Ernst angle, we chose to run a $10^{\circ} \mathrm{FA}$ for most of the experiments. Practically, there is little difference in running $8^{\circ}, 10^{\circ}$ or $12^{\circ}$. Clearly, a small FA is preferred at high field since it keeps the specific absorption rate (SAR) low. On the other hand, the contrast is likely to be best at angles higher than the Ernst angle and this is particularly true for the case of inflowing blood. The problem with too high a flip angle is that the signal from the arteries becomes suppressed further along the direction of flow. A lower flip angle creates a more spin density weighted sequence. From this perspective, the magnitude image is basically a low flip angle, long echo TOF sequence which has reduced sensitivity to pulsation artifacts $(29,30)$. The scan time for each FA was 8 minutes.

Third, we considered the issue of echo time. For a left-handed system, the phase can be written as

$$
\Delta \varphi=\gamma \Delta B T
$$


where $\Delta \mathrm{B}$ represents the local field deviation caused by the differences in magnetic susceptibility between tissues. To optimize the CNR, we used the standard deviation for the phase noise

$$
\sigma_{\text {phase }}=\frac{1}{S N R_{\text {mag }}}=\frac{\sigma_{\text {mag }}}{\text { Signal }}
$$

yielding

$C N R_{\text {phase }}=\frac{\Delta \varphi}{\sigma_{\text {phase }}}=\frac{\gamma \cdot \Delta B \cdot T E \cdot \text { Signal }_{\text {mag }}}{\sigma_{\text {mag }}}=\frac{\gamma \cdot \Delta B \cdot T E \cdot K \cdot e^{\frac{-T E}{T 2^{*}}}}{\sigma_{\text {mag }}}$

where $\mathrm{K}$ is a parameter that includes the $\mathrm{T} 1$, spin density and flip angle dependent part of the signal. The optimization of this CNR with respect to TE then yields TE $=\mathrm{T} 2 *$. Allowing a shorter echo time to scan faster, and holding the imaging time constant (i.e., for the case of $\mathrm{CNR}_{\text {pput }}=C N R / \sqrt{\text { imagingtime }}$ where imaging time varies with $\mathrm{TE}$ ), the CNR per square root imaging time becomes:

$$
C N R_{\text {phase }}=\frac{\gamma \cdot \Delta B \cdot \sqrt{T E} \cdot K \cdot e^{\frac{-T E}{T 2^{*}}}}{\sigma_{\text {mag }}}
$$

and the optimization of $\mathrm{CNR}_{\mathrm{pput}}$ yields $\mathrm{TE}=0.5 \cdot \mathrm{T} 2 *$. We chose to use the latter value which (along with choosing a roughly in-phase water/fat echo time) yielded TE $=15.6 \mathrm{~ms}$. This long echo time, compared to the usual short echo TOF approach, also made it possible to keep a low bandwidth of 80 to $100 \mathrm{~Hz} /$ pixel. A longer echo time of $\sim 30 \mathrm{~ms}$, to match T2*, would yield significant aliasing in the phase from local magnetic field effects. For tissues with calcium, there would be little signal left. The CNR of the phase between vessel wall and arterial lumen with $\mathrm{TE}=0.5 \cdot \mathrm{T} 2 *$, compared to $\mathrm{TE}=\mathrm{T} 2 *$, was found to be equal to 0.823 of the peak CNR with the longer echo time.

Two different echo times were collected in one healthy subject and estimates for $\mathrm{T} 2 *$ were made for vessel wall, adjacent muscle and venous blood. The scan time for each TE was 8 minutes. The SWI sequence was run twice with different echo times, once with $7.38 \mathrm{~ms}$ and once with $12.3 \mathrm{~ms}$, both roughly in-phase relative to fat, in the axial plane, with full flow compensation and with a $3^{\circ}$ flip angle to avoid pulsation artifacts. The pixel-by-pixel signal was too noisy to calculate a T2* image accurately. To overcome this, a region-of-interest (ROI) was drawn to cover a portion of the vessel wall in a series of 9 slices. Average vesselwall ROI size in each slice was about 370 voxels. T2* was calculated by taking the natural logarithm of the ratio of the signals measured from two echo times for a region of interest. For instance, a region around the adjacent muscle was drawn (in the most homogeneous part of the muscle) with an average ROI size of 6000 pixels in each slice and $\mathrm{T} 2 *$ was evaluated. Similarly, T2* of venous blood was calculated with an average venous lumen ROI size of 1900 voxels in each slice. A single echo scan with a flip angle of $2^{\circ}$ and TE $7.38 \mathrm{~ms}$ was also run to assess the relative spin densities of vessel-wall, muscle and venous blood.

\section{Vessel Wall Magnitude and Phase Measurements}

To achieve clear delineation between vessel wall, lumen and surrounding tissue, and to allow reformatting of the sagittal data into the transverse plane or orthogonal to the long axis of the vessel without blurring, we collected the sagittal data with $0.5 \mathrm{~mm}$ isotropic resolution 
for 5 healthy volunteers with an acquisition time of 16 minutes. The phase of the vessel wall was measured taking an ROI in the sagittal data in different locations. Despite the variations in the magnitude signal due to the variable flip angle at the edge of the coil, the vessel wall phase could still be reliably measured in these regions (since only the SNR of the phase is affected the phase itself when the magnitude signal varies). Therefore, ROIs from the top to the of the visible portions of the femoral artery were taken. The lumen SNR and muscle SNR calculated on both magnitude and phase images. The contrast-to-noise ratio of vessel wall/lumen and vessel wall/muscle were also calculated.

\section{Patient Studies}

Eighteen patients (11 male, 7 female, range: $39-79$, mean age 65 ) with symptomatic lower extremity peripheral arterial disease (PAD) were scanned. The study was approved by our Institutional Review Board and informed consent was obtained from all patients. All 18 patients underwent 64-detector computed tomography (LightSpeed VCT, GE Healthcare, United States) before participating in this study. The scan protocol was as follows: $64 \mathrm{~mm} \times 0.625 \mathrm{~mm}$ collimation, interval $0.625 \mathrm{~mm}, 120 \mathrm{kv}, 420 \mathrm{~mA}$, pitch 1.375 , tablefeed $110 \mathrm{~mm}$, with a $512 \times 512$ matrix size. The MDCT scanning ranged from the upper pole of the patella to the fibular head. Multiplanar reconstructions (MPR) and maximum intensity projections (MIP) were done in standard sagittal planes, with a slice thickness of $1.0 \mathrm{~mm}$ and reconstruction increment of $1.0 \mathrm{~mm}$. For the MRI scans, SWI was acquired on a 3.0T wholebody scanner (Trio; Siemens Healthcare, Erlangen, Germany). An 8 channel transmit/ receive knee coil was used and data were acquired centered on the popliteal trifurcation with sagittal slices. A high resolution SWI scan (taking 8 minutes) was run on each leg of the subjects. The imaging parameters for this sequence were: $\mathrm{TE}=15.6 \mathrm{~ms}, \mathrm{TR}=25 \mathrm{~ms}, \mathrm{FA}=$ $10^{\circ}$ or $12^{\circ}$, resolution $=0.5 \mathrm{~mm} \times 0.5 \mathrm{~mm} \times 1 \mathrm{~mm}$, with an acquisition matrix of $512 \times 384 \times$ 64. For phase processing, the central $64 \times 64 \mathrm{k}$-space points were zero-filled to $512 \times 512$ for each slice and a low-resolution complex image was created. This image was then complex-divided into the original image to remove low spatial frequency phase behavior and its associated aliasing.

\section{Calcification Measurements}

On CT images, the attenuation threshold value of $130 \mathrm{HU}$ was used to identify calcification. The distance between calcifications and femoral or tibial bones and the area of calcification was manually measured by experienced radiologist. On phase images, the vessel wall of femoral or tibial artery was first identified. The location of the calcification was then identified as the area of higher diamagnetism on the vessel wall. On the magnitude images, the location of the calcification was determined as the area of hypo intense on the vessel wall. SPIN software (Detroit, MI) was used to interpolate the images by a factor of 4 and measure the boundary of the calcifications. The correlation of calcification area (CA) between CT and MR images was performed and a Pearson correlation coefficient calculated. A p-value of less than 0.05 was considered to be statistically significant. The agreement of CA measurements by MR and CT was assessed by using the Bland and Altman plot. Quantitative variables were expressed as mean value \pm standard deviation.

\section{RESULTS}

The two-echo scans revealed that the muscle and vessel wall had basically the same tissue properties in terms of $\mathrm{T} 2 *$ and spin density (see Table 1 ). The $\mathrm{T} 2 *$ of venous blood was also similar to that in muscle, and all measured T2* values were found to be in good agreement with literature values for similar tissues (31-34). The $\Delta \mathrm{TE}$ chosen between the two echoes was not sufficient to estimate $\mathrm{T} 2 *$ for arterial blood, but another paper estimated the $\mathrm{T} 2 *$ of arterial blood to be $72 \mathrm{~ms}$ (35). The T1 values for the muscle and blood were taken from the 
literature (36) (see Table 1). Using the described optimal experimental parameters and the tissue parameters given in Table 1, the expected SNR and CNR between arterial vessel wall, arterial and venous blood signals were calculated and plotted in Figure 1. Since the muscle and vessel wall had almost equal T2* values, we assumed their $\mathrm{T} 1$ value to be equal to $1412 \mathrm{~ms}$ for simulation purposes. The simulations also took the $\mathrm{T} 2 *$ of the veins to be $27.3 \mathrm{~ms}$ and the arteries to be $72 \mathrm{~ms}$. The magnitude images for different flip angles are shown in Figure 2. The femoral artery has the highest SNR throughout the image for a flip angle of $10^{\circ}$, which is close to the Ernst angle for muscle. Although the upper part of the image shows a better SNR for the femoral artery at $15^{\circ}$, by the middle of the leg, the signal is already saturated. The most uniform signal comes from the $5^{\circ}$ flip angle but the signal is only $2 / 3$ of that from the $10^{\circ}$ flip angle (Table 2). Signal ratios of vessel wall/muscle, arterial blood/muscle and venous blood/muscle, averaged over 9 slices, were evaluated and the spin density of muscle was fixed at 80 (arbitrary units) to obtain the spin density values for the vessel-wall and venous blood relative to muscle.

The results of SNR and CNR measurements for 5 healthy volunteers are summarized inTable 3. (All phase measurements were made in the center of a given slice.) The phase contrast between vessel lumen and vessel wall was $\Delta \varphi=234+/-33$ in Siemens phase units (spu) or 0.359 radians. This gives a CNR of 7:1 for arterial wall to vessel lumen. It is possible to quote this phase value in terms of a field deviation $\delta$ in "parts per million" (ppm) using the relationship $\Delta \varphi \approx 4 \pi \delta$ in radians for an echo time of $15.6 \mathrm{~ms}$. Therefore $\delta=$ $0.0287 \mathrm{ppm}$ for the arterial vessel wall with respect to arterial blood. Figure 3 shows an example image of normal arterial and vessel wall in both magnitude and phase. In the magnitude images, signal variations in the lower popliteal artery are due to the varying flip angle at the edge of the coil. However on the phase images, vessel wall could still be reliably measured in these regions.

A total of 19 calcifications in the femoral vessel wall were identified with SWI in 8 subjects. The mean area of calcification measured on CT, magnitude and phase images was $0.37 \pm 0.17 \mathrm{~cm}^{2}, 0.29 \pm 0.13 \mathrm{~cm}^{2}, 0.38 \pm 0.18 \mathrm{~cm}^{2}$ respectively. The Pearson correlation coefficient of the measured lesion area between CT and magnitude image is $0.85(\mathrm{p}<0.001)$; between CT and phase image is $0.92(\mathrm{p}<0.001)$ (Figure 4). CA measured on SWI images were plotted using the Bland-Altman method (Figure 5). A typical case having popliteal artery calcification is shown in Figure 6. Both magnitude and phase images show the calcifications clearly in the popliteal artery wall and correlate well with the CT image. A second case is shown in Figure 7 with a much smaller calcification. The positive phase representing the diamagnetic nature of the calcification is shown as well as a clear representation of the lumen/wall contrast showing a variable thickness vessel wall.

\section{DISCUSSION}

There has been previous work in quantifying calcifications using different kinds of MRI techniques(37-39). However, in their work phase imaging was not typically used. In the current study, we proposed the use of SWI to image vessel lumen and vessel wall because it offers a unique contrast in the phase images that makes it possible to both visualize vessel wall and recognize signal from calcifications. One of the surprising findings was that the arterial vessel wall had a susceptibility of $0.0287 \mathrm{ppm}$, not accounting for geometric factors, in the field change (i.e., treating the phase shift as a chemical shift). Although most tissue is, in fact, diamagnetic, this shows for the first time local differences in diamagnetism between vessel wall and the lumen of the vessel and surrounding tissue. The calcium deposits tended to have a much higher diamagnetism, sometimes as high as 10 times that of the vessel wall. This means that there is a broad range of calcium concentrations that could be visualized before the phase from calcium would become indistinguishable from that of vessel wall. The 
change in phase of the vessel wall also means that with the resolution being used in these experiments that it is easy to separate artery from vein because the vessel wall has a distinct contrast to the blood signal in the arteries or veins.

Earlier work in PAD involved in vitro images with intravascular ultrasound $(40,41)$, intravascular MRI (42) or with black-blood imaging. The closest approach to what we used here is actually conventional time-of-flight MR angiography (MRA). We used a much lower flip angle than that used in MRA since we do not require a TOF method to get good contrast. The $5^{\circ}$ flip angle images have good contrast for the femoral artery. This can be understood by examining the predicted contrast in the presence of no flow (see Figure 1), where the optimal flip angle for artery wall contrast is just higher than $5^{\circ}$. However, overall, the $10^{\circ} \mathrm{FA}$ gave a higher signal throughout the central part of the leg and we chose this value for the general scanning. We relied on a fully flow compensated sequence, with low flip angle to suppress pulsatility artifacts (low flip angles lead to a more spin density weighted image) and a sagittal acquisition which also suppresses signal variability by saturating incoming spins. Earlier papers involving PAD were also done at $0.5 \mathrm{~T}$ and $1.5 \mathrm{~T}$ and were acquired either with TOF MRA or black-blood methods. In fact, the images from Meissner et al. (18) already are beginning to look like the magnitude images we have here, although not with quite the same resolution, contrast or echo time. Still, in their work, the contrast at $1.5 \mathrm{~T}$ is good enough to see plaques in the femoral wall.

Previous approaches (particularly for carotid artery imaging) had required special surface coils, fat saturation, rapidly flowing blood, venous saturation, thick slices, and provided limited coverage. Even then, the normal vessel wall did not appear to be well delineated in many publications, although thick diseased vessel wall was well seen with these techniques. Our work requires no surface coils and the sequence can be run with $0.25 \mathrm{~mm}^{3}$ voxel resolution in only 8 minutes without the use of parallel imaging. The images show good vessel wall/lumen contrast; no fat saturation or venous saturation is required. Moreover, there is little dependence on blood flow for either patent or stenosed vessels, and the phase can help differentiate calcium from vessel wall.

Optimizing the SNR for TE has to be traded off with the choice of an in-phase signal relative to fat. SWI is usually used with a very long echo time of $20 \mathrm{~ms}$ at $3 \mathrm{~T}$ to enhance the venous phase. This was not necessary here and, in principle, if there is enough diamagnetic contrast present in the vessel wall and enough signal to differentiate it from the surrounding blood and tissue, an echo time even shorter than $15.6 \mathrm{~ms}$, could be used. However, given the SNR we have in the experiment so far, there is already a rather broad profile to the vessel wall phase. On the other hand, despite the reduced signal that might be present for vessel wall contrast, a short echo would have more signal from the regions with calcium and less aliasing from the calcium. One possible solution to this would be to use a double echo sequence (43) with a high bandwidth in the first echo and a low bandwidth for the second echo still at $15.6 \mathrm{~ms}$. The first echo would have reduced SNR but the phase from calcium would still be visible.

The phase images provide a new means to identify vessel wall that can have a distinct advantage over the magnitude images. In the magnitude images, it is easy to see the lumen/ wall boundary but unless there is a low signal region beside the outside of the vessel (which is the case in some areas of the vessel), the surrounding tissue (muscle for example) looks the same and the boundary of the outer vessel wall can be lost. Further, there can be other tissues at the edges of the vessel wall that may appear dark mimicking calcium, but in fact are not calcium. Such an example is shown in Figure 3, where the phase image shows the vessel wall in tact in that area which appears dark in the magnitude image. 
The CNR between vessel wall and lumen was found to be 7.4:1 in the phase images and 11.7:1 in the magnitude images. That is good enough to allow further reduction in scan time using parallel imaging or increasing the resolution, both of which will cause a loss in SNR of about $\sqrt{2}$ leaving roughly a 5:1 CNR ratio. That may still be good enough for clinical imaging purposes and more work needs to be done in that direction. Although this paper has not focused on the vessel wall of veins or distinguishing arteries from veins, there is evidence that we can see venous wall as well and previous papers have already shown that the phase can be used to distinguish veins from background tissue (44).

One problem that currently exists is for a complicated plaque (Figure 7). In that case, the phase may not appear with a uniform phase enhancement because of a very complicated field dipole effect. On the other hand, a smaller more point-like calcification will clearly show the cloverleaf-like dipole effect. This dipole effect is partly removed by the current high pass phase filter but not completely and not for smaller structures. In fact, it can be used as an easy means to identify calcium (45). Newer methods that process the phase to produce susceptibility maps may help resolve these problems (46). The proper processing of the phase images will lead to a source image whose sign will immediately reveal whether the source is calcium or blood products for example. It is also possible to do the forward problem for a given shape and look for a sign match of the phase behavior. The appearance of the phase can also be complicated by the voxel aspect ratio (47). The dipole effect also causes a blooming in the phase images explaining why the area in the phase image for the calcification is larger than that in the magnitude image. Ironically, we find that the correlation of calcified area is better for the phase than the magnitude images. This may be related to partial volume effects in both CT and MR, as well as the fact that CT may overestimate the calcified volume. The partial volume effects in phase are a bit different than those in the magnitude image. In the latter case, the edges of the vessel wall may have only a fraction of the signal in the center of the wall, while in the former case, the phase is independent of the magnitude making the vessel wall appear possibly two pixels wider.

Measurement of the size of the calcification and its distance to other structures shows that the same calcification seen in MDCT is clearly depicted in the both the magnitude and phase images at the same place and with roughly the same size. The correlation between CT and MRI is very good, giving us better confidence in our interpretation that SWI does not only replicate the CT findings but also shows that the effect is from calcium. The phase images, however, could correlate generally better with CT for a different reason. It is quite possible that small amounts of calcium can be seen with phase that cause little or no signal loss but still manifest as a change in phase. This requires further investigation but given the results shown here where strong changes in phase of nearly $\pi$ radians are seen when the magnitude signal loss is intermediate to high then an effect that is 10 times smaller would still be seen on the phase images but not on the magnitude images.

Future work with this method will be to study a broader range of patients looking for general PAD, pushing the resolution to $250 \mu$ in plane, and testing the methodology on carotid arteries. We anticipate that SWI will play a complementary role to the current multi-contrast approach in studying atherosclerosis.

\section{Acknowledgments}

Grant Support:

This work was supported in part by the National Institutes of Health Grant 2R01HL 62983-04 and the State of Michigan Grant 085P5200251. 


\section{REFERENCES}

1. Yuan C, Kerwin WS. MRI of atherosclerosis. J Magn Reson Imaging. 2004; 19(6):710-719. [PubMed: 15170778]

2. Saam T, Ferguson MS, Yarnykh VL, et al. Quantitative evaluation of carotid plaque composition by in vivo MRI. Arteriosclerosis, thrombosis, and vascular biology. 2005; 25(1):234-239.

3. Underhill HR, Yarnykh VL, Hatsukami TS. Carotid plaque morphology and composition: initial comparison between 1.5- and 3.0-T magnetic field strengths. Radiology. 2008; 248(2):550-560. [PubMed: 18574135]

4. Bornstedt A, Bernhardt P, Hombach V, et al. Local excitation black-blood imaging at 3T: application to the carotid artery wall. Magn Reson Med. 2008; 59(5):1207-1211. [PubMed: 18421686]

5. Chu B, Phan BA, Balu N, Yuan C, Brown BG, Zhao XQ. Reproducibility of carotid atherosclerotic lesion type characterization using high resolution multicontrast weighted cardiovascular magnetic resonance. J Cardiovasc Magn Reson. 2006; 8(6):793-799. [PubMed: 17060101]

6. Finn JP, Edelman RR. Black-blood and segmented k-space magnetic resonance angiography. Magnetic resonance imaging clinics of North America. 1993; 1(2):349-357. [PubMed: 7584229]

7. Stary HC, Chandler AB, Dinsmore RE, et al. A definition of advanced types of atherosclerotic lesions and a histological classification of atherosclerosis. A report from the Committee on Vascular Lesions of the Council on Arteriosclerosis, American Heart Association. Circulation. 1995; 92(5): 1355-1374. [PubMed: 7648691]

8. Shinnar M, Fallon JT, Wehrli S, et al. The diagnostic accuracy of ex vivo MRI for human atherosclerotic plaque characterization. Arteriosclerosis, thrombosis, and vascular biology. 1999; 19(11):2756-2761.

9. Eubank W, Yuan C, Fisher E, Luna J. Endarterectomy specimen shrinkage: comparison of T2weighted MR imaging of specimen ex vivo to histological processes. J Vasc Interv Radiol. 1998; 4(161):161-170.

10. Helft G, Worthley SG, Beygui F, et al. [Identification of unstable coronary atherosclerotic plaques]. Archives des maladies du coeur et des vaisseaux. 2001; 94(6):583-590. [PubMed: 11480156]

11. Wasserman BA, Smith WI, Trout HH 3rd, Cannon RO 3rd, Balaban RS, Arai AE. Carotid artery atherosclerosis: in vivo morphologic characterization with gadolinium-enhanced double-oblique MR imaging initial results. Radiology. 2002; 223(2):566-573. [PubMed: 11997569]

12. Steinman DA, Rutt BK. On the nature and reduction of plaque-mimicking flow artifacts in blackblood MRI of the carotid bifurcation. Magn Reson Med. 1998; 39(4):635-641. [PubMed: 9543426]

13. Hatsukami TS, Ross R, Polissar NL, Yuan C. Visualization of fibrous cap thickness and rupture in human atherosclerotic carotid plaque in vivo with high-resolution magnetic resonance imaging. Circulation. 2000; 102(9):959-964. [PubMed: 10961958]

14. Parker DL, Yuan C, Blatter DD. MR angiography by multiple thin slab 3D acquisition. Magn Reson Med. 1991; 17(2):434-451. [PubMed: 2062215]

15. Koktzoglou I, Chung YC, Carroll TJ, Simonetti OP, Morasch MD, Li D. Three-dimensional blackblood MR imaging of carotid arteries with segmented steady-state free precession: initial experience. Radiology. 2007; 243(1):220-228. [PubMed: 17392255]

16. Koktzoglou I, Li D. Diffusion-prepared segmented steady-state free precession: Application to 3D black-blood cardiovascular magnetic resonance of the thoracic aorta and carotid artery walls. $\mathrm{J}$ Cardiovasc Magn Reson. 2007; 9(1):33-42. [PubMed: 17178678]

17. Criqui MH, Fronek A, Barrett-Connor E, Klauber MR, Gabriel S, Goodman D. The prevalence of peripheral arterial disease in a defined population. Circulation. 1985; 71(3):510-515. [PubMed: 3156006]

18. Meissner OA, Rieger J, Rieber J, et al. High-resolution MR imaging of human atherosclerotic femoral arteries in vivo: validation with intravascular ultrasound. J Vasc Interv Radiol. 2003; 14(2 Pt 1):227-231. [PubMed: 12582191] 
19. Boos M. Clinical value of high resolution MRI of vessel wall lesions in peripheral atherosclerosis disease: first in vivo experience before and after PTA. MAGMA. 1996; 127:94.

20. Wyttenbach R, Gallino A, Alerci M, et al. Effects of percutaneous transluminal angioplasty and endovascular brachytherapy on vascular remodeling of human femoropopliteal artery by noninvasive magnetic resonance imaging. Circulation. 2004; 110(9):1156-1161. [PubMed: 15326071]

21. Isbell DC, Meyer CH, Rogers WJ, et al. Reproducibility and reliability of atherosclerotic plaque volume measurements in peripheral arterial disease with cardiovascular magnetic resonance. $\mathrm{J}$ Cardiovasc Magn Reson. 2007; 9(1):71-76. [PubMed: 17178683]

22. Yarnykh VL, Yuan C. Multislice double inversion-recovery black-blood imaging with simultaneous slice reinversion. J Magn Reson Imaging. 2003; 17(4):478-483. [PubMed: 12655588]

23. Guzman RJ, Brinkley DM, Schumacher PM, Donahue RM, Beavers H, Qin X. Tibial artery calcification as a marker of amputation risk in patients with peripheral arterial disease. Journal of the American College of Cardiology. 2008; 51(20):1967-1974. [PubMed: 18482666]

24. Qiao JH, Doherty TM, Fishbein MC, et al. Calcification of the coronary arteries in the absence of atherosclerotic plaque. Mayo Clinic proceedings. 2005; 80(6):807-809. [PubMed: 15948300]

25. Pentecost MJ, Criqui MH, Dorros G, et al. Guidelines for peripheral percutaneous transluminal angioplasty of the abdominal aorta and lower extremity vessels. A statement for health professionals from a special writing group of the Councils on Cardiovascular Radiology, Arteriosclerosis, Cardio-Thoracic and Vascular Surgery, Clinical Cardiology, and Epidemiology and Prevention, the American Heart Association. Circulation. 1994; 89(1):511-531. [PubMed: 8281692]

26. Wright LB, Matchett WJ, Cruz CP, et al. Popliteal artery disease: diagnosis and treatment. Radiographics. 2004; 24(2):467-479. [PubMed: 15026594]

27. Haacke EM, Mittal S, Wu Z, Neelavalli J, Cheng YC. Susceptibility-weighted imaging: technical aspects and clinical applications, part 1. AJNR Am J Neuroradiol. 2009; 30(1):19-30. [PubMed: 19039041]

28. Mittal S, Wu Z, Neelavalli J, Haacke EM. Susceptibility-weighted imaging: technical aspects and clinical applications, part 2. AJNR Am J Neuroradiol. 2009 (IN PRESS)..

29. Haacke, EM.; Brown, RW.; Thompson, MR.; Venkatesan, R. Magnetic Resonance Imaging: Physical Principles and Sequence Design. 1st edition. Wiley-Liss; 1999 June 15. 1999.

30. Haacke EM, Xu Y, Cheng YC, Reichenbach JR. Susceptibility weighted imaging (SWI). Magn Reson Med. 2004; 52(3):612-618. [PubMed: 15334582]

31. O'Regan DP, Callaghan MF, Fitzpatrick J, Naoumova RP, Hajnal JV, Schmitz SA. Cardiac T2* and lipid measurement at 3.0 T-initial experience. Eur Radiol. 2008; 18(4):800-805. [PubMed: 18034347]

32. Vandenborne K, Walter G, Ploutz-Snyder L, Dudley G, Elliott MA, De Meirleir K. Relationship between muscle $\mathrm{T} 2 *$ relaxation properties and metabolic state: a combined localized $31 \mathrm{P}$ spectroscopy and 1H-imaging study. Eur J Appl Physiol. 2000; 82(1-2):76-82. [PubMed: 10879446]

33. O'Connor JP, Naish JH, Jackson A, et al. Comparison of normal tissue R1 and R*2 modulation by oxygen and carbogen. Magn Reson Med. 2009; 61(1):75-83. [PubMed: 19097212]

34. Blockley, NP.; Francis, ST.; Gowland, PA. Dependence of R2* on oxygenation and contrast agent concentration in human blood at 3T. Seattle: 2006. p. 2516

35. Zhao JM, Clingman CS, Narvainen MJ, Kauppinen RA, van Zijl PC. Oxygenation and hematocrit dependence of transverse relaxation rates of blood at 3T. Magn Reson Med. 2007; 58(3):592-597. [PubMed: 17763354]

36. Stanisz GJ, Odrobina EE, Pun J, et al. T1, T2 relaxation and magnetization transfer in tissue at 3T. Magn Reson Med. 2005; 54(3):507-512. [PubMed: 16086319]

37. Kucharczyk W, Henkelman RM. Visibility of calcium on MR and CT: can MR show calcium that CT cannot? Ajnr. 1994; 15(6):1145-1148. [PubMed: 8073985]

38. Herzka, D.; Nezafat, R.; Rahmer, J.; Manning, W.; Boernert, P. Ultra-short TE Imaging Protocol for Detection of Aortic Calcification. Toronto: 2008 April. p. 947 
39. Herzka, D.; Rahmer, J.; Nezafat, R.; Chan, R.; Liu, W.; Boernert, P. High-resolution Ultra-short TE Imaging of ex vivo Human Carotid Plaques Correlates with CT. Toronto: 2008 April. p. 962

40. Yock PG, Linker DT, Angelsen BA. Two-dimensional intravascular ultrasound: technical development and initial clinical experience. J Am Soc Echocardiogr. 1989; 2(4):296-304. [PubMed: 2697308]

41. Nissen SE, Yock P. Intravascular ultrasound: novel pathophysiological insights and current clinical applications. Circulation. 2001; 103(4):604-616. [PubMed: 11157729]

42. Omary RA, Green JD, Schirf BE, Li Y, Finn JP, Li D. Real-time magnetic resonance imagingguided coronary catheterization in swine. Circulation. 2003; 107(21):2656-2659. [PubMed: 12756160]

43. Du YP, Jin Z. Simultaneous acquisition of MR angiography and venography (MRAV). Magn Reson Med. 2008; 59(5):954-958. [PubMed: 18429022]

44. Wang Y, Yu Y, Li D, et al. Artery and vein separation using susceptibility-dependent phase in contrast-enhanced MRA. J Magn Reson Imaging. 2000; 12(5):661-670. [PubMed: 11050635]

45. Wu Z, Mittal S, Kish K, Yu Y, Hu J, Haacke EM. Identification of calcification with MRI using susceptibility-weighted imaging: a case study. J Magn Reson Imaging. 2009; 29(1):177-182. [PubMed: 19097156]

46. Haacke EM, Cheng NY, House MJ, et al. Imaging iron stores in the brain using magnetic resonance imaging. Magnetic resonance imaging. 2005; 23(1):1-25. [PubMed: 15733784]

47. Xu Y, Haacke EM. The role of voxel aspect ratio in determining apparent vascular phase behavior in susceptibility weighted imaging. Magn Reson Imaging. 2006; 24(2):155-160. [PubMed: 16455403] 

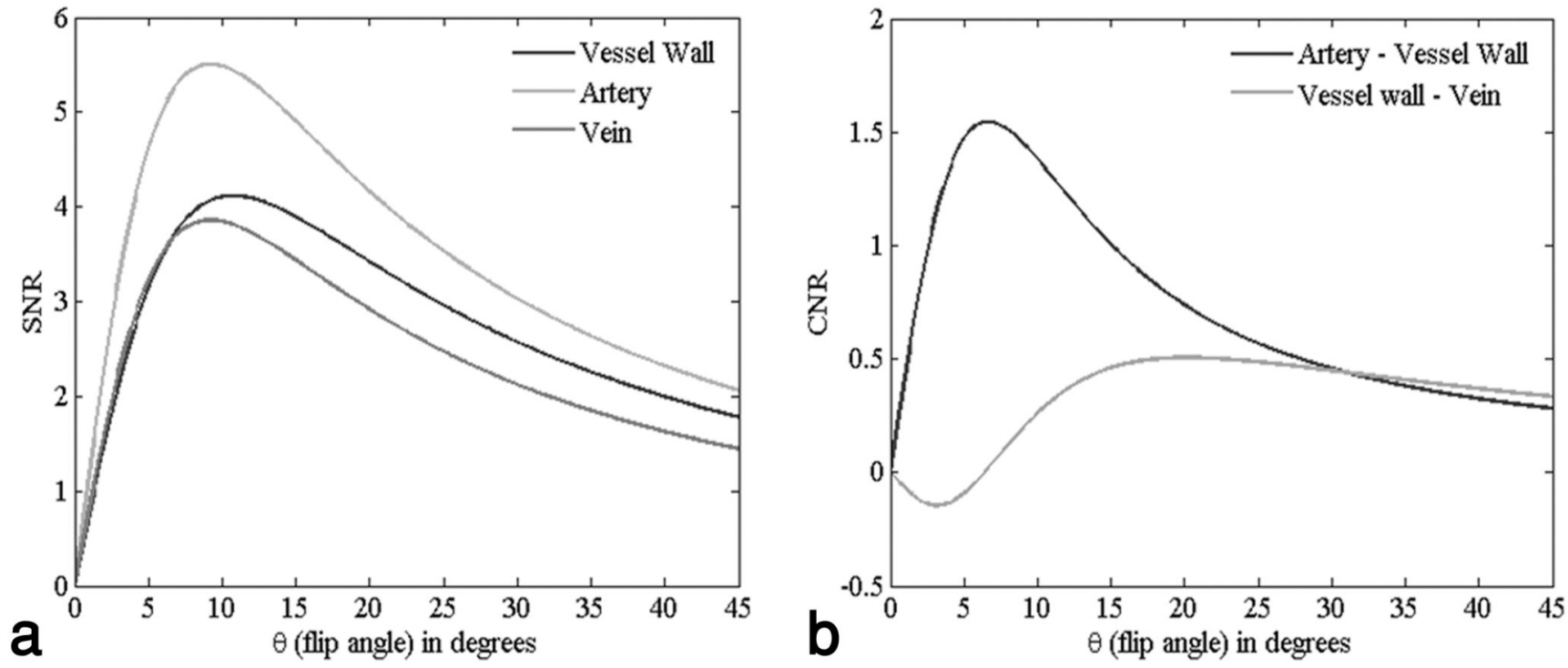

Figure 1.

Theoretical plots of (a) SNR and (b) CNR for the vessel wall and blood in arbitrary units. T1 of both arterial and venous blood is assumed to be $1932 \mathrm{~ms}$. T1 of vessel wall is assumed to be $1412 \mathrm{~ms}$. No time-of-flight effort is included in these simulations. 

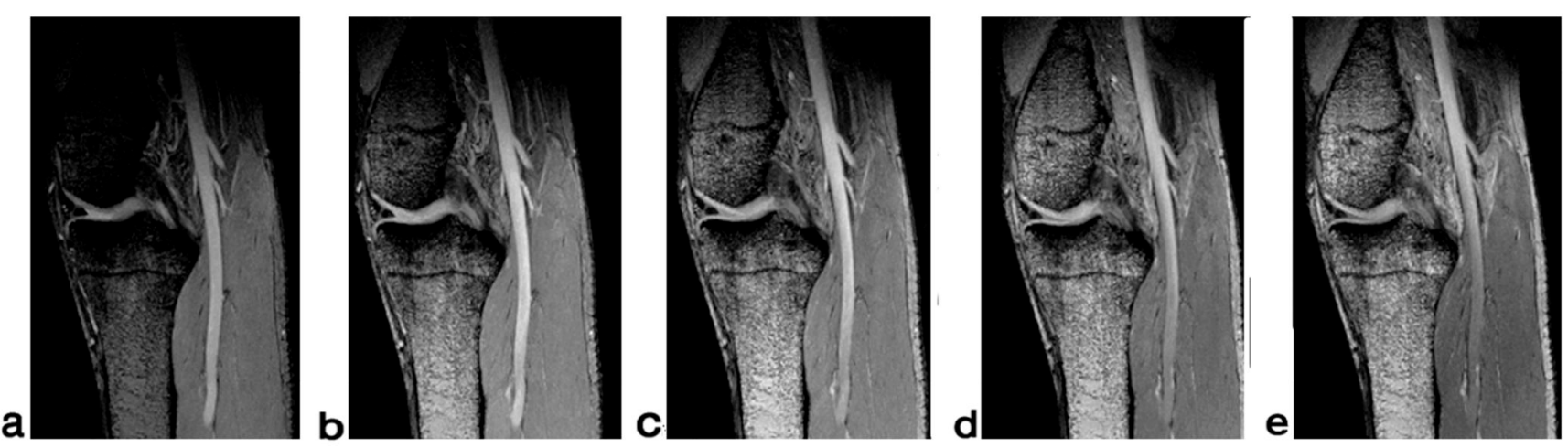

Figure 2.

Magnitude images of the knee from a healthy volunteer using different flip angles. From left to right, the FAs are: (a) $5^{\circ}$, (b) $10^{\circ}$, (c) $15^{\circ}$, (d) $20^{\circ}$, and (e) $25^{\circ}$. The femoral artery is best depicted with a flip angle of $10^{\circ}$. Although the $5^{\circ}$ result shows the most uniform signal, the highest signal is obtained from the $10^{\circ}$ flip angle. The arterial signal in the higher flip angle images becomes more and more saturated. 

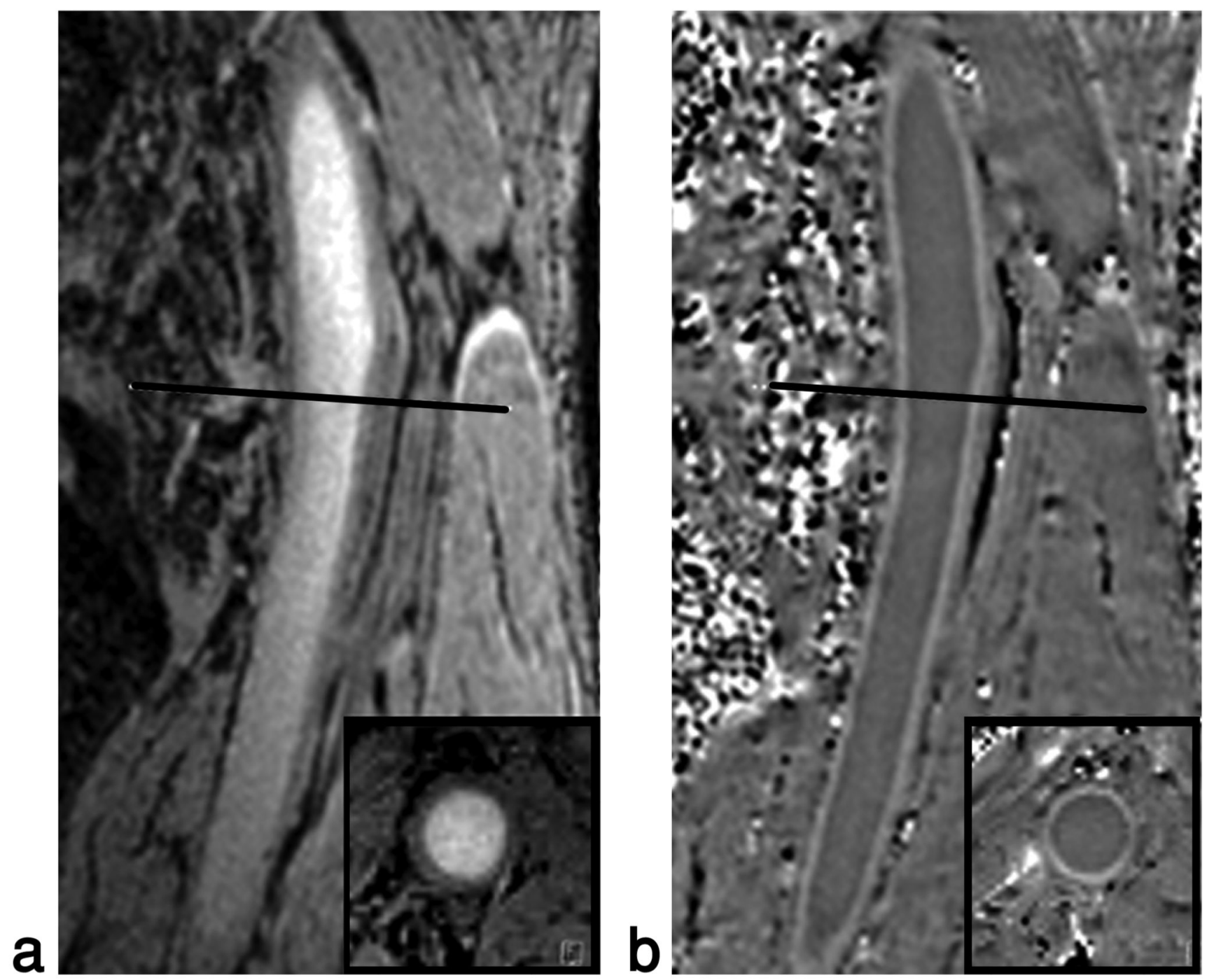

Figure 3.

Sagittal magnitude (a) and SWI filtered phase (b) images. The reformatted transverse images, created through a plane cutting, are shown as inserts in the bottom right of each image. Note the dark area in the magnitude that might be mistaken for calcium whereas on the phase image the vessel wall is clearly seen with no calcium in this area. The cross section of the vessel and vessel wall are well seen. Data was acquired with $0.5 \mathrm{~mm}$ isotropic resolution in a healthy volunteer. 

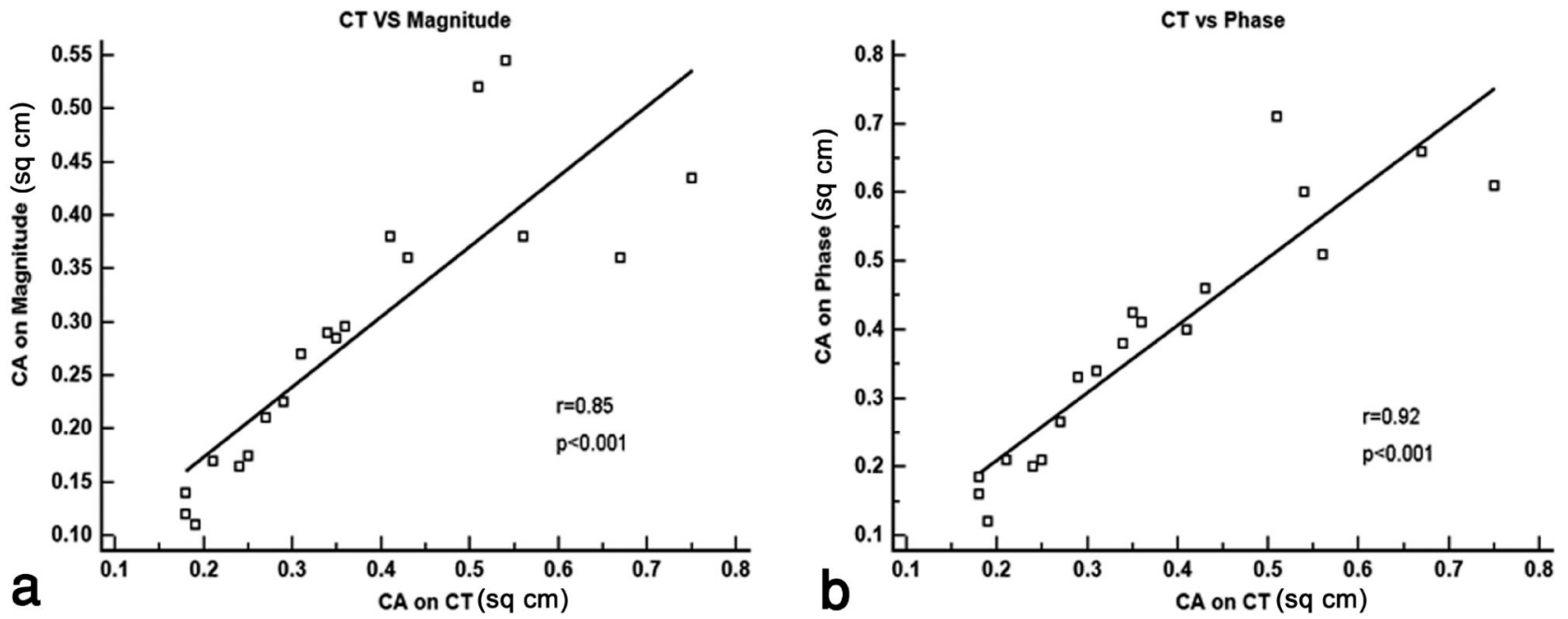

Figure 4.

Correlation between calcification area (CA) on CT versus that on SWI, both (a) magnitude and (b) phase. 

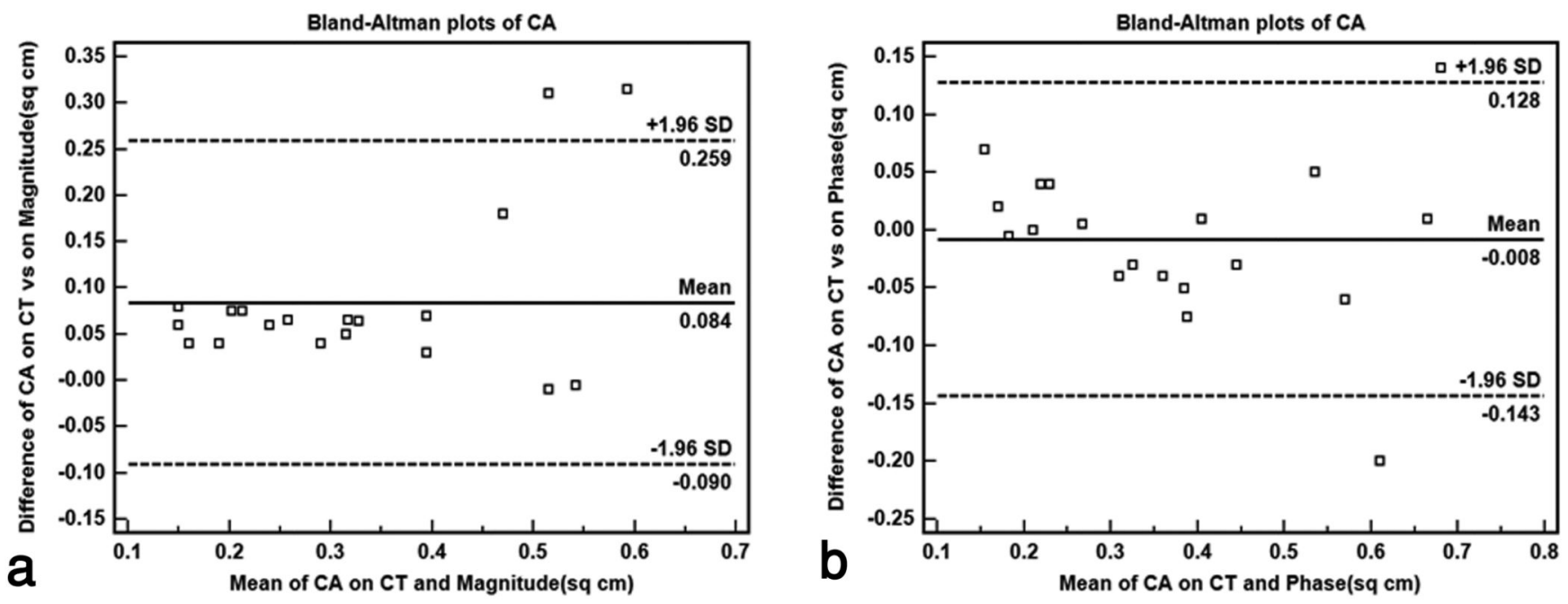

Figure 5.

Bland-Altman plots of calcification area (CA) correlation between CT and SWI, both (a) magnitude and (b) phase. 

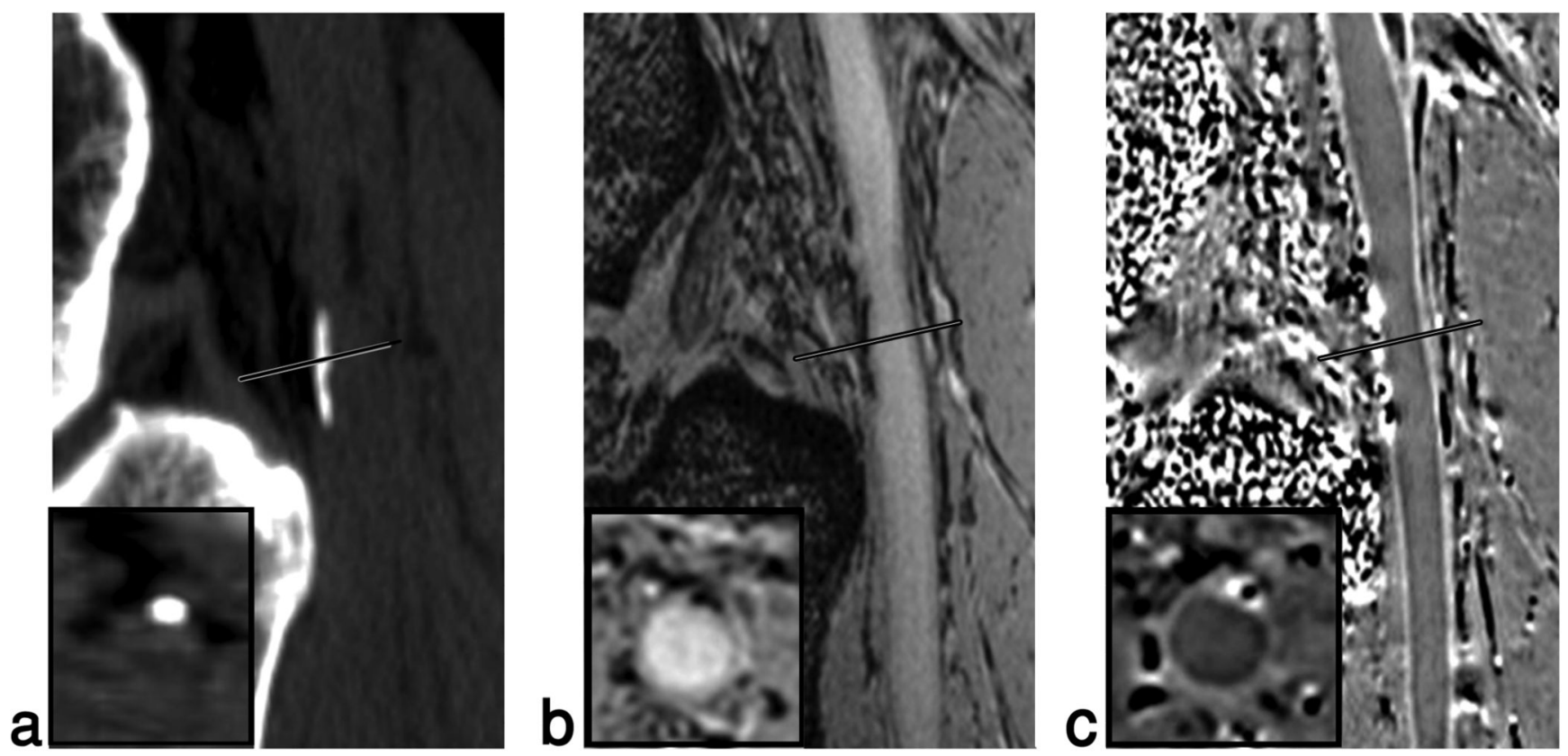

Figure 6.

(a) CT scan showing calcification at the edge of the popliteal artery just behind the knee. (b) Magnitude gradient echo image showing the signal loss from the calcification in the same area. (c) Phase image showing the diamagnetic effect from the calcification. Note the similar shape and extent of the calcification in both the CT and MR results. Inserts are zoomed images of the cross section of the vessels in reformatted transverse images. 

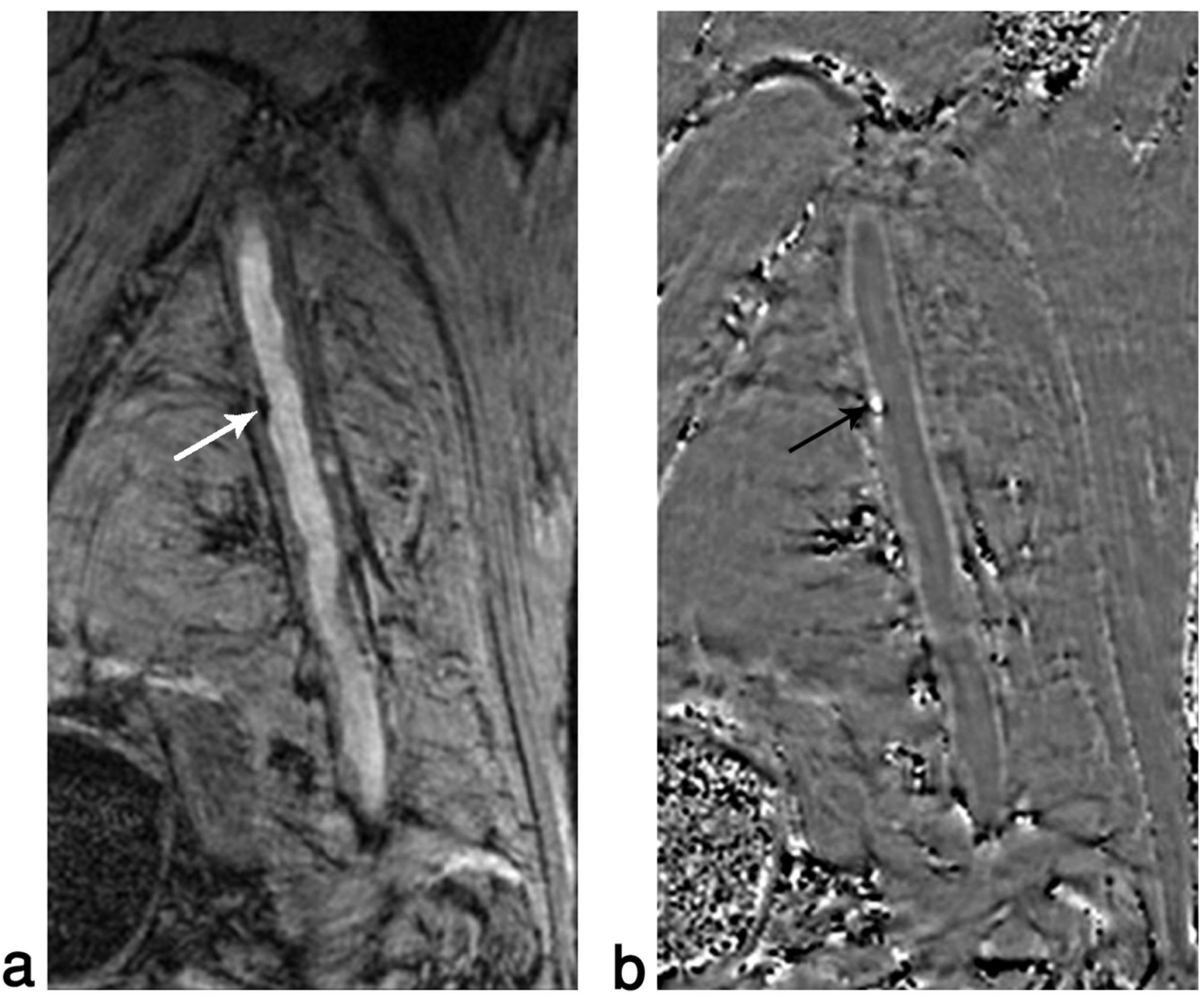

Figure 7.

Delineation of femoral artery wall in a case with variable wall thickening and a single calcification. Magnitude image (a) shows the lumen bright which serves as a good marker between the inside wall and blood. The phase image (b) shows the vessel wall with a brighter than background signal indicating that the vessel wall is diamagnetic. The calcification also shows as a small white enhancement. 
Table 1

Tissue properties used in the simulations

\begin{tabular}{lllll}
\hline & Spin Density $\boldsymbol{a}$ & T2* & $\begin{array}{l}\text { Standard } \\
\text { deviation } \\
\text { of T2* (in ms) }\end{array}$ & T1 \\
\hline Vessel Wall & 83 & 24.4 & 3.15 & $1412^{b}$ \\
Muscle & 80 & 25.9 & 1.64 & $1412^{c}$ \\
Vein & 85 & 24.3 & 2.26 & $1932^{c}$ \\
Artery & 85 & $72^{b}$ & - & $1932^{b}$ \\
\hline
\end{tabular}

${ }^{a}$ Values taken from the low flip angle measurements

${ }^{b}$ Values taken from the literature

${ }^{c} \mathrm{~T} 1$ values assumed to be equal for arterial and venous blood and also equal for vessel wall and muscle. 


\section{Table 2}

Effect of FA on magnitude images

\begin{tabular}{lll}
\hline FA & SNR & $\begin{array}{l}\text { Standard } \\
\text { deviation }\end{array}$ \\
\hline $5^{\circ}$ & 26.7 & 3.5 \\
$10^{\circ}$ & 39.7 & 5.5 \\
$15^{\circ}$ & 34.5 & 7.2 \\
$20^{\circ}$ & 29.9 & 4.3 \\
$25^{\circ}$ & 24.4 & 4.4 \\
\hline
\end{tabular}

FA=flip angle 


\section{Table 3}

Vessel wall measurements on 5 volunteers

\begin{tabular}{lll}
\hline & Magnitude & Phase \\
\hline $\mathrm{SNR}_{\text {wall }}$ & $20.6 \pm 7.0$ & - \\
$\mathrm{SNR}_{\text {lumen }}$ & $32.3 \pm 11.7$ & - \\
$\mathrm{CNR}_{\mathrm{W} / \mathrm{L}}$ & $11.7 \pm 6.1$ & $7.4 \pm 2.4$ \\
$\mathrm{CNR}_{\mathrm{W} / \mathrm{M}}$ & $4.3 \pm 5.6$ & $6.6 \pm 2.4$ \\
\hline
\end{tabular}

$\mathrm{CNRW} / \mathrm{L}=$ the contrast to noise ratio between vessel wall and lumen, $\mathrm{CNRW} / \mathrm{M}=$ contrast to noise ratio between vessel wall and muscle. 